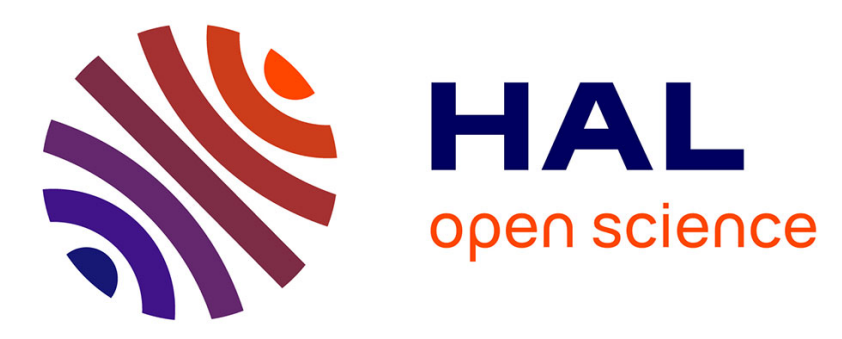

\title{
Editorial Social cognitive systems in smart environments: Approaches for learning, reasoning, and adaptation
}

Amir Aly, Shashank Pathak, Sean Andrist, Armando Tacchella

\section{- To cite this version:}

Amir Aly, Shashank Pathak, Sean Andrist, Armando Tacchella. Editorial Social cognitive systems in smart environments: Approaches for learning, reasoning, and adaptation. Cognitive Systems Research, 2019, 58, pp.230-233. 10.1016/j.cogsys.2019.07.006 . hal-02264979v2

HAL Id: hal-02264979

https://hal.science/hal-02264979v2

Submitted on 28 Aug 2019

HAL is a multi-disciplinary open access archive for the deposit and dissemination of scientific research documents, whether they are published or not. The documents may come from teaching and research institutions in France or abroad, or from public or private research centers.
L'archive ouverte pluridisciplinaire HAL, est destinée au dépôt et à la diffusion de documents scientifiques de niveau recherche, publiés ou non, émanant des établissements d'enseignement et de recherche français ou étrangers, des laboratoires publics ou privés. 


\section{Editorial \\ Social Cognitive Systems in Smart Environments: \\ Approaches for Learning, Reasoning, and Adaptation}

Amir Aly, Shashank Pathak, Sean Andrist, Armando Tacchella

\section{Contents}

1. Bunlon, F. and Gazeau, J-P. and Colloud, F. and Marshall, P. J. and Bouquet, C. A., "Joint action with a virtual robotic vs human agent".

2. Perula-Martinez, R. and Castro-Gonzalez, A. and Malfaz, M. and AlonsoMartin, F. and Salichs, M. A., "Bioinspired decision-making for a socially interactive robot".

3. Nauta, J. and Mahieu, C. and Michiels, C. and Ongenae, F. and De Backere, F. and De Turck, F. and Khaluf, Y. and Simoens, P., "Proactive positioning of a social robot intervening upon behavioral disturbances of persons with dementia in a smart nursing home".

4. Ropero, F., Vaquerizo, D., Pablo Muoz, P., Barrero, D., R-Moreno, M., "LARES: An AI-based teleassistance system for emergency home monitoring".

5. Maamar, Z., "Weaving Cognition into the Internet-of-Things: Application to Water Leaks".

6. Garcia-Aunon, P. and Roldan, J-J. and Barrientos, A., "Monitoring traffic in future cities with aerial swarms: developing and optimizing a behavior-based surveillance algorithm".

7. Veronese, L., Badue, C., Cheein, F., Jose Guivant, J., De Souza, A., "A Single Sensor System for Mapping in GNSS-denied Environments".

8. Wilson, G. and Pereyda, C. and Raghunath, N. and De la Cruz, G. and Nesaei, S. and Minor, B. and Schmitter-Edgecombe, M. and Taylor, M. 
and Cook, D., "Robot-Enabled Support of Daily Activities in Smart Home Environments".

9. Guerrero, E. and Lu, M-H. and Yueh, H-P. and Lindgren, H., "Designing and Evaluating an Intelligent Augmented Reality System for Assisting Older Adults' Medication Management".

\section{Introduction}

Robots with social competencies are no longer confined to controlled laboratory environments as research prototypes. They are beginning to appear in the real world as informational guides in airports, museums, and hospitals, collaborating closely alongside humans on factor floors, and assisting families in the home. As robots move into these new spaces, they must grapple with challenging and dynamic environments, requiring them to learn and adapt over time to unforeseen situations. The most important features of these environments are the humans that these robots need to work with and alongside, requiring them to learn and adapt specifically to these humans' cognitive and behavioral characteristics.

The use of robots side by side with cognitive science and artificial intelligence allows for modeling the hypotheses and theories of cognition so as to provide a clear conceptualization for cognitive functions considering the effect of the smart environment. This opens the door to several applications that require intelligent behaviors, such as: reducing cognitive load for individuals interacting with a smart environment, assisting the elderly and people with cognitive disabilities and disorders in mobility and daily tasks, and developing a cloud-connected robot system to incrementally share knowledge between robots in different smart environments about the behavioral characteristics of human users in order to interact with them adaptively. To meet the requirements of these target applications, robots need to be able to learn how to use the capabilities of their smart environments to better address the needs of the human users.

This special issue aims at shedding light on the intersection of artificial intelligence and cognitive robotics from the theoretical and technical aspects covering the basic research and its applications. The recent advances and the future scope of cognitive robotics including the new methodologies and applied technologies are principal topics in this special issue. 


\section{Summary of the special issue}

The concept of "social robots" was coined by Dautenhahn and Billard [1], which refers to endowing embodied agents with high-level cognitive functions to engage in social interaction with human users $[2,3,4]$. Developing such intelligent agents takes robotics research to the center of artificial intelligence, human-robot interaction, neuroscience, and cognitive psychology research so as to cross the distance from human intelligence to artificial intelligence, where the fundamental target is to make robots able to perceive, reason, and behave adaptively and rationally in different contexts of interaction $[5,6,7]$.

Among the many anticipated roles for robots in the future is that of supporting various human activities in a substantial way. Aside from all the technological hurdles that have to be overcome with respect to hardware and control to make robots fit to various tasks, the added complication is that humans have many conscious and subconscious expectations, and thus supporting humans is mostly a cognitive rather than physical coordination activity. This introduces new challenges for the AI and robotics community and requires fundamental changes to the traditional approach to the design of autonomy.

During the last decades, researchers in both artificial intelligence and cognitive science were involved in developing theories of cognition to serve in building computational models that can make robots able to interact with human users in an intelligent manner in smart environments (e.g., smart home, smart city, etc.), such as: situated and embodied cognition [8, 9], perceptual symbol systems [10], and working memory [11], which could give interesting insights into understanding the way humans think and behave. These computational models are critical for enabling robots to interact with and reason about the human users in their environments. However, generic models of human behavior and cognition must be augmented with techniques for learning and adapting over time, as real-world scenarios contain far more complexities than could ever be feasibly anticipated by researchers and designers. Robots will need to navigate unforeseen physical environments, carry out unforeseen tasks, and interact with humans that behave in unforeseen ways in order to be robust and effective.

When faced with challenges of uncertain and unobservable environments, using artificial intelligence enables to overcome parts of it. For example, through the implementation of simultaneous localization and mapping (SLAM), a robot is able to navigate successfully in a world not known a-priori [12], 
and the same is true even in the presence of humans. However, if the model of uncertainty is assumed for human behavior, more efficient artificial intelligence strategies can be designed. On one hand AI can assist human strategies (such as in mixed decision making [13]) and human behavior (such as in advanced driver assistance systems), and on the other hand, the better understanding of human intentions can help proactive and efficient planning as demonstrated by Kwon and Suh [14]. This synergy between the human and the robot has been an emerging theme lately. For example, Unhelkar et al. [15] pose it as a co-navigation problem where anticipating human behavior is the key, while the work of Nikolaidis et al. [16] is an example of how the adaptation is a two-way process. It is, therefore, highly desirable to seek greater synergy between the AI methods and human-centric robotics approaches.

In this special issue, we take a step forward towards bridging between different disciplines of cognitive science, artificial intelligence, and robotics research with the objective of shedding light on current and future challenges in cognitive intelligent robotics in smart environments. By smart environments, we imply not just the cases where the environment is fitted with smart-sensor, but also where the intelligence is rooted in an intelligent device working in close co-ordination with the human. This special issue includes the following research studies:

Bunlon et al. [17] discuss how humans share a task with a non-human agent (a virtual human and a robotic agent), and explore the joint Simon effect in light of the influence of prior experience with the agent on performance. Moreover, the study investigates whether the Simon effect is affected by sensorimotor experience during which a participant manipulates the robotic agent through an exoskeleton.

Perula-Martinez et al. [18] propose a decision making system for social robots that drives their actions taking into account both the user and the robot states. The system is based on bio-inspired concepts, such as motivations, drives, and well-being that could facilitate the rise of natural behaviors to ease the acceptance of a robot by users.

Nauta et al. [19] investigate the way to position a robot in a nursing home, taking into account the profile and location of the residents who exhibit behavioral disturbances so as to provide an adequate support tool for caregivers. The proposed algorithm minimizes the time between the detection of a behavioral disturbance and having the robot arrived near to a resident on one side, and starting an interaction scenario on the other side. 
In this context of robot and nursing, Ropero et al. [20] present an overall system of elderly-care through efficient usage of recent developments in artificial intelligence and in wireless sensor network technologies. In particular, they employ efficient wireless communication for interpreting the needs of an elder through a robot which then harnesses tele-operation to meet those needs. Their anomaly detection and subsequent answering of the needs was found to be general enough in two separate cases of elderly homes, observed through several weeks.

Maamar [21] examines "Thing" empowerment in the Internet-of-Things (IoT) paradigm. Specifically, the contribution focuses on adding cognitive capabilities that enable "Things" to change their predefined work flow to adapt to changing environments. The mechanism is based on an integration of the IoT paradigm with Business Processes (BP) descriptions. The framework is applied to a real-world case study about detection of leaks in a water distribution system.

Wilson et al. [22] discuss how smart environments offer valuable technologies for activity monitoring and health assessment with a specific reference to integration of robots into smart environments in order to provide more interactive support of individuals with functional limitations. They describe their system RAS (Robot Activity Support) system which partners smart environment sensing, object detection and mapping, and robot interaction to detect and assist with activity errors that may occur in everyday settings.

Guerrero et al. [23] present a research methodology towards developing intelligent assistive systems that utilize augmented reality for tracking and distributing prescribed medicines for older adults in a home health care scenario. Their multi-faceted methodology brings together first-order logical proofs, activity theory, and targeted user studies.

Garcia-Aunon et al. [24] focus on the problem of traffic monitoring, which is a key issue to develop smarter and more sustainable cities in the future. Their work presents an aerial swarm that continuously monitors the traffic in a simulated, but realistic city. The control algorithm of the aerial swarm is based on six behaviors with twenty-three parameters that must be tuned. The optimization of parameters is carried out with a genetic algorithm in a simplified and faster simulator.

de Paula Veronese et al. [25] look into the problem of accurate localization of autonomous vehicles in the absence of GNSS (Global Navigation Satellite System). They harness recent developments in LiDAR technology as well as SLAM (Simultaneous Localization And Mapping) and through the assump- 
tion of navigation on a road in the map, they perform efficient single-sensor localization.

\section{Acknowledgments}

The guest editors would like to thank the authors whose contributions allowed this special issue to succeed. Similarly, the guest editors would like to sincerely thank the reviewers who kindly provided very helpful reviews for the submitted papers to this special issue.

\section{References}

[1] K. Dautenhahn, A. Billard, Bringing up robots or-the psychology of socially intelligent robots: From theory to implementation, in: Proceedings of the Third Annual Conference on Autonomous Agents.

[2] A. Aly, A. Tapus, Towards an online fuzzy modeling for human internal states detection, in: Proceedings of the 12th International Conference on Control Automation Robotics and Vision (ICARCV), Guangzhou,China, pp. 1563-1570.

[3] A. Aly, Towards an interactive human-robot relationship: Developing a customized robot's behavior to human's profile, Ph.D. thesis, ENSTA ParisTech, France, 2014.

[4] A. Aly, A. Tapus, An online fuzzy-based approach for human emotions detection: An overview on the human cognitive model of understanding and generating multimodal actions, in: S. Mohammed, J. Moreno, K. Kong, Y. Amirat (Eds.), Intelligent Assistive Robots - Recent Advances in Assistive Robotics for Everyday Activities, Series: Springer Tracts in Advanced Robotics (STAR), volume 106, Springer International Publishing, Switzerland, 2015, pp. 185-212.

[5] P. Norvig, S. Russell, Artificial intelligence: A modern approach, Prentice Hall, USA, 2010.

[6] A. Aly, A. Tapus, An integrated model of speech to arm gestures mapping in human-robot interaction, in: Proceedings of the 14th IFAC Symposium on Information Control Problems in Manufacturing (INCOM), Bucharest, Romania. 
[7] A. Aly, A. Tapus, Multimodal adapted robot behavior synthesis within a narrative human-robot interaction, in: Proceedings of of the IEEE/RSJ International Conference on Intelligent Robots and Systems (IROS), Hamburg, Germany.

[8] J. Brown, A. Collins, S. Duguid, Situated cognition and the culture of learning, Educational Researcher 18 (1989) 32-42.

[9] F. Varela, E. Rosch, E. Thompson, The embodied mind: Cognitive science and human experience, MIT Press, USA, 1991.

[10] S. Harnad, The symbol grounding problem, Physica D 42 (1990) 335346.

[11] A. Baddeley, G. Hitch, Working memory, in: G. Bower (Ed.), The Psychology of Learning and Motivation, Academic Press, USA, 1974.

[12] S. Thrun, W. Burgard, D. Fox, Probabilistic Robotics, MIT Press, USA, 2005.

[13] S. Li, J. Z. Li, Hybridising human judgment,AHP, simulation and a fuzzy expert system for strategy formulation under uncertainty, Expert Systems with Applications 36 (2009) 5557-5564.

[14] W. Y. Kwon, I. H. Suh, Planning of proactive behaviors for humanrobot cooperative tasks under uncertainty, Knowledge-Based Systems 72 (2014) 81-95.

[15] V. V. Unhelkar, C. Prez-D'Arpino, L. Stirling, J. A. Shah, Human-robot co-navigation using anticipatory indicators of human walking motion, in: Proceedings of the IEEE International Conference on Robotics and Automation (ICRA), Seattle, WA, USA.

[16] S. Nikolaidis, S. Nath, A. D. Procaccia, S. Srinivasa, Game-theoretic modeling of human adaptation in human-robot collaboration, in: Proceedings of the 12th ACM/IEEE International Conference on HumanRobot Interaction (HRI), Vienna, Austria.

[17] F. Bunlon, J.-P. Gazeau, F. Colloud, P. J. Marshall, C. A. Bouquet, Joint action with a virtual robotic vs human agent, Cognitive Systems Research (2019). 
[18] R. Perula-Martinez, A. Castro-Gonzalez, M. Malfaz, F. Alonso-Martin, M. A. Salichs, Bioinspired decision-making for a socially interactive robot, Cognitive Systems Research (2019).

[19] J. Nauta, C. Mahieu, C. Michiels, F. Ongenae, F. D. Backere, F. D. Turck, Y. Khaluf, P. Simoens, Pro-active positioning of a social robot intervening upon behavioral disturbances of persons with dementia in a smart nursing home, Cognitive Systems Research (2019).

[20] F. Ropero, D. Vaquerizo, P. Muoz, D. F. Barrero, M. D. R-Moreno, LARES: An AI-based teleassistance system for emergency home monitoring, Cognitive Systems Research (2019).

[21] Z. Maamar, Weaving cognition into the Internet-of-Things: Application to water leaks, Cognitive Systems Research (2019).

[22] G. Wilson, C. Pereyda, N. Raghunath, G. de la Cruz, S. Nesaei, B. Minor, M. Schmitter-Edgecombe, M. Taylor, D. Cook, Robot-enabled support of daily activities in smart home environments, Cognitive Systems Research (2019).

[23] E. Guerrero, M.-H. Lu, H.-P. Yueh, H. Lindgren, Designing and evaluating an intelligent augmented reality system for assisting older adults' medication management, Cognitive Systems Research (2019).

[24] P. Garcia-Aunon, J. J. Roldn, A. Barrientos, Monitoring traffic in future cities with aerial swarms: Developing and optimizing a behavior-based surveillance algorithm, Cognitive Systems Research (2019).

[25] L. de Paula Veronese, C. Badue, F. A. Cheein, J. Guivant, A. F. D. Souza, A single sensor system for mapping in GNSS-denied environments, Cognitive Systems Research (2019). 